\title{
Inverse Association Between lodine Status and Prevalence of Metabolic Syndrome: A Cross-Sectional Population-Based Study in a Chinese Moderate lodine Intake Area
}

\author{
Jing Zhao ${ }^{1,2, *}$ \\ Yinbiao $\mathrm{Su}^{3, *}$ \\ Jin-An Zhang ${ }^{2}$ \\ Ming Fang ${ }^{4}$ \\ Xuerong $\mathrm{Liu}^{2}$ \\ $\mathrm{Xi} \mathrm{Jia}^{2}$ \\ Xinming $\mathrm{Li}^{1,4}$
}

'Graduate School, Shanghai University of Traditional Chinese Medicine, Shanghai, 201203, People's Republic of China; ${ }^{2}$ Department of Endocrinology, Shanghai University of Medicine \& Health Sciences Affiliated Zhoupu Hospital, Shanghai, 201318, People's Republic of China; ${ }^{3}$ Department of Endocrinology, Jinshan Hospital of Fudan University, Shanghai, 201508, People's Republic of China; ${ }^{4}$ Department of Cardiology, Shanghai University of Medicine \& Health Sciences Affiliated Zhoupu Hospital, Shanghai, 201318, People's Republic of China

*These authors contributed equally to this work

Correspondence: Xinming Li Graduate School, Shanghai University of Traditional Chinese Medicine, Department of Cardiology, Shanghai University of Medicine \& Health Sciences Affiliated Zhoupu Hospital, Shanghai, 201318, People's Republic of China

$\mathrm{Tel}+86$ 2I-68I35590-3160

Email XinmingLi-SH@hotmail.com
Purpose: To investigate the relationship between iodine intake status and the prevalence of metabolic syndrome (MetS) through a population-based survey.

Patients and Methods: In total, 2691 Chinese adults participated in this cross-sectional study, and they were stratified by urinary iodine concentration (UIC) and sex. Fasting blood samples were used to assess biochemical parameters, including thyroid function and antibodies. Urine samples were collected in the morning to measure UIC. Multivariate regression logistic analysis was performed for the overall population and sex subgroups.

Results: An inverse association was observed between iodine intake status and MetS prevalence in Chinese adults. Compared with individuals with adequate iodine status, those with high-iodine status had significantly low MetS risks, and the adjusted odds ratios (95\% confidence interval) were $0.70(0.57-0.86, P<0.01)$ and $0.75(0.6-0.95, P<0.05)$. A high MetS risk was observed in the iodine-deficient group, which did not reach statistical significance. There was a significant inverse linear trend between the risk of MetS and UIC in the total population and male subgroup $(P$ for trend $<0.05)$, which was not observed in the female subgroup ( $P$ for trend $>0.05$ ).

Conclusion: An inverse association was observed between iodine intake status and the risk of developing MetS in Chinese adults. Sufficient iodine status is a potential protective factor for MetS development. Males may benefit from increased iodine intake, while females would need to achieve a more-than-adequate iodine status to gain metabolic benefits.

Keywords: dyslipidemia, hypertension, hyperglycemia, abdominal obesity

\section{Introduction}

Metabolic syndrome (MetS) is a combination of interrelated metabolic risk factors that include larger waist circumference (WC), high blood pressure (BP), dyslipidemia, and elevated fasting glucose levels. ${ }^{1}$ Therefore, MetS increases the risk of CVD, T2D, specific cancers, ${ }^{2}$ and total mortality. Parallel with the cosmopolitan prevalence of nutrition transition, MetS has become a pandemic in both adults and adolescents. ${ }^{3}$ MetS prevalence in Chinese adults was reported to be $33.9 \%$, with approximately 454 million people being affected. ${ }^{4}$ MetS imposes excessive burden on patients and the health care system in terms of medical, social, and economic aspects. Consequently, there has been an urgent need for effective interventions that could reduce the incidence of MS and that are economically feasible. The etiology of MetS 
includes a multiplex interaction among genetic, environmental, and metabolic factors. ${ }^{5,6}$ Changes in dietary habits have also been flagged as potential drivers of the increasing prevalence of metabolic disorders. ${ }^{7}$ Therefore, identifying associated factors could prevent or delay the occurrence of MetS in individuals.

Iodine is an essential micronutrient required for optimal growth, development, metabolism, and neurocognitive function in humans. Baumann first identified the role of thyroidconcentrated iodine as early as 124 years ago. In vivo, a large amount of iodine is used to synthesize thyroid hormones, which play an essential role in normal growth, neurological function, development, and various metabolic processes. ${ }^{8}$ Appropriate intake of dietary iodine is critical for sustaining normal thyroid function. ${ }^{9,10}$ Previous studies elucidated the association between thyroid abnormalities and metabolic disturbances, ${ }^{11,12}$ even in the euthyroid population with only positive thyroid antibodies. ${ }^{13}$ Several studies conducted in rodent models with metabolic disorders also observed the protective effect of iodine. ${ }^{14,15}$ Researches have shown that metabolic disorders are closely related to iodine nutritional status in adults. A cross-sectional study conducted in US adults demonstrated that low urinary iodine concentration (UIC) was associated with increased incidence of dyslipidemia. ${ }^{16}$ In addition, studies from other countries also elaborated that patients with type 2 diabetes had lower dietary iodine intake ${ }^{17}$ and lower UICs than controls. ${ }^{18}$ However, Liu et al reported that excessive iodine intake may be associated with elevated BP and hyperglycemia and decreased high-density lipoprotein cholesterol (HDLC) levels, but increasing rates of hypertension and dyslipidemia were not observed. ${ }^{19}$

Studies have confirmed that MetS prevalence is strongly related to regional economic and existed variation based on geographical locations. ${ }^{4}$ However, epidemiological surveys on the relationship between iodine intake status and the prevalence of MetS are scarce. Therefore, this cross-sectional study aimed to assess the correlation between iodine intake status and the prevalence of MetS.

\section{Subjects, Materials and Methods Participants}

In total, 2824 Chinese individuals who were residents of the Jinshan District of Shanghai were randomly recruited for this cross-sectional study in 2016, after determining the required sample size. ${ }^{20}$ Participants from the Chinese Han population, who were aged $\geq 18$ years, and had resided in the Jinshan
District of Shanghai for 5 years were included; we excluded pregnant women. Among the participants, 27 used iodinecontaining drugs, and 105 used intravenous iodinated contrast agents during the last 3 months prior to sample collections; therefore, they were not considered eligible for inclusion. Furthermore, one individual was excluded owing to incomplete data. Finally, 2691 individuals (1315 males and 1376 females) were eligible and finally enrolled (Figure 1).

The Medical Ethics Committee of China Medical University approved the study procedures, and we adhered to the 1964 Helsinki Declaration and its later amendments (NO.AF-SOP-07-1.0-01). All participants provided written informed consent.

\section{Clinical Examination and Laboratory Testing}

The questionnaire was administered to each participant by trained staff members. The questionnaire was used to collect information on age, sex, education level, income, smoking, type of salt intake, salt intake/day, medical condition, and medication history. Experienced physicians measured each individual's height, weight, WC, BP, and other indicators between 7 a.m. and 9 a.m., while in their fasting state. Smokers were defined as those who were currently smoking. Body mass index (BMI) was calculated as weight $(\mathrm{kg}) /$ height $^{2}\left(\mathrm{~m}^{2}\right)$.

Fasting blood samples were used to measure fasting plasma glucose (FPG), total cholesterol (TC), TG, HDL-C, low-density lipoprotein cholesterol (LDL-C), and serum uric acid (UA) levels using an automatic analyzer (BS-180, Mindray), according to an enzymatic method. Thyroid function and thyroid antibodies were assessed using the electrochemiluminescence immunoassays with a Cobas 601 analyzer (Roche Diagnostic, Switzerland), which included the detection of thyroid-stimulating hormone (TSH) levels, presence of thyroid peroxidase antibody (TPOAb), and presence of thyroglobulin antibody $(\mathrm{TgAb})$. Urinary samples were collected in the morning to measure the UIC using inductively coupled plasma mass spectrometry (Agilent 7700x; Agilent Technologies). All biospecimens were collected between 7 a.m. and 10 a.m. and stored at $-20^{\circ} \mathrm{C}$ until transportation to the central laboratory for uniformity in detecting clinical variables.

\section{Diagnostic Methods for MetS}

MetS was diagnosed according to the criterion of the Joint Interim Statement (JIS) of the NHLBI/AHA/WHF/IAS/ 


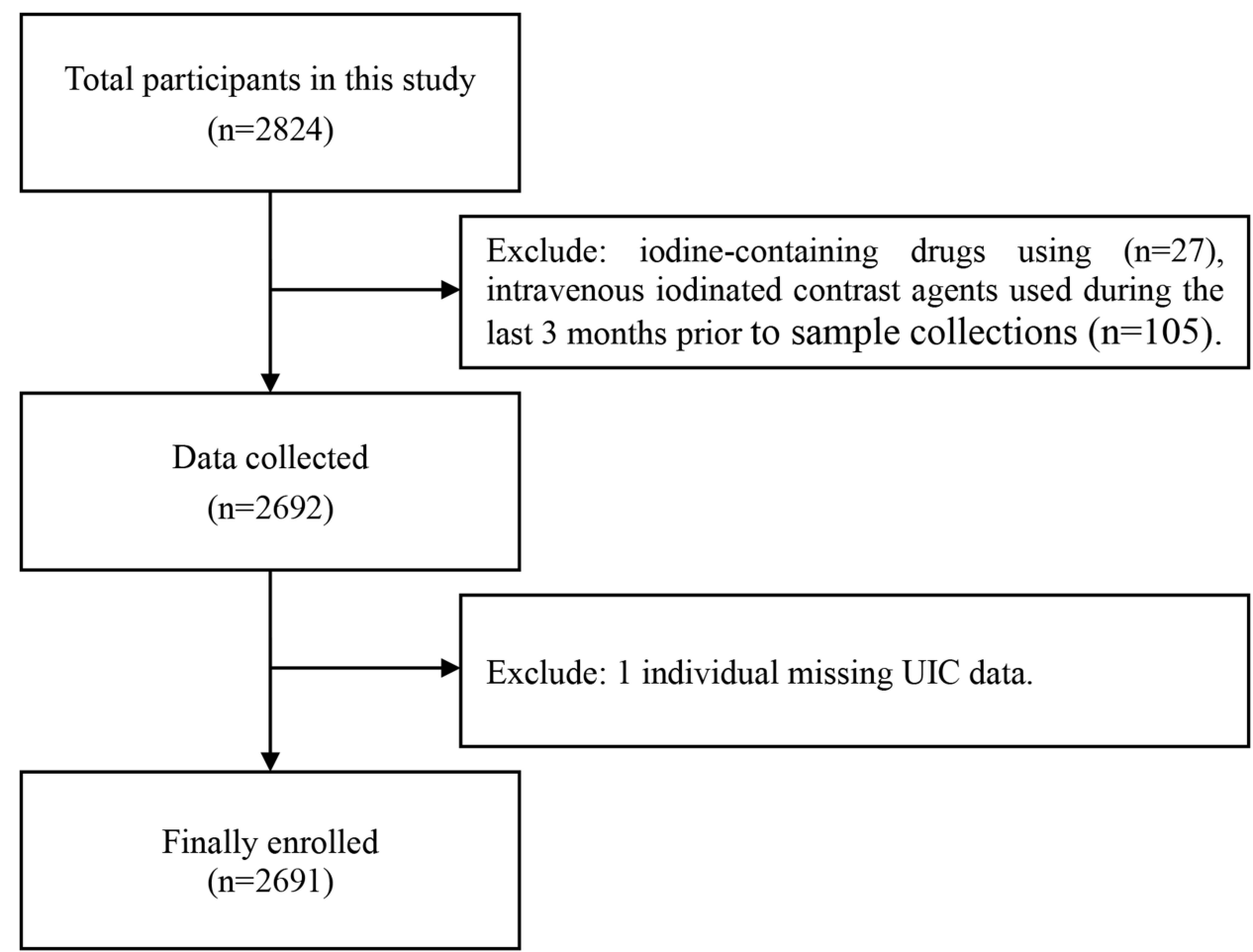

Figure I Flowchart of participants.

IAS when three or more of the followings factors were identified in an individual: WC (according to the population- and country-specific definition for China) $\geq 85 \mathrm{~cm}$ (male) or $80 \mathrm{~cm}$ (female); systolic BP (SBP) $\geq 130$ $\mathrm{mmHg}$ and/or diastolic BP (DBP) $\geq 85 \mathrm{mmHg}$ or history of high BP or use of BP-lowering medication; HDL-C $<1.0 \mathrm{mmol} / \mathrm{L}$ (male) or $<1.3 \mathrm{mmol} / \mathrm{L}$ (female) or ongoing drug treatment to reduce HDL-C; TG $\geq 1.7 \mathrm{mmol} / \mathrm{L}$ or use of TG-lowering drugs; FPG $\geq 100 \mathrm{mg} / \mathrm{dL}$ or ongoing glucose-lowering drug treatment. ${ }^{1}$

\section{Category Criteria for lodine Status}

As the most suitable biochemical marker, UIC directly reflects the dietary iodine intake and is commonly used worldwide. In this study, we referred to the UIC standards recommended by the World Health Organization (WHO) ${ }^{21}$ and then divided all participants into three groups depending on their UIC values: iodine-deficient group, UIC $<100$ $\mu \mathrm{g} / \mathrm{L}$; iodine-adequate group, UIC between 100 and 199 $\mu \mathrm{g} / \mathrm{L}$; and high-iodine group, $\mathrm{UIC} \geq 200 \mu \mathrm{g} / \mathrm{L}$.

\section{Statistical Analysis}

Continuous variables are presented as mean \pm Standard deviation or median and interquartile range (IQR). Differences between two groups of continuous variables were analyzed using the $T$-test or Mann-Whitney $U$-test. Categorical variables are presented as number and percentage. Chi-square test was used for inter-group comparisons. Additionally, trend tests were fulfilled to detect discrepancies in characteristics according to the UIC groups. Trends in continuous variables were evaluated using "nptrend" in STATA, and trends in dichotomous categorical variables were assessed using the Cochran-Armitage test. The prevalence of the MetS and its parameters was described as frequency with $95 \%$ confidence interval (CI). Trend test among the UIC groups was based on assigning the median of each subgroup as the variable value.

First, univariate logistic regression was performed to confirm the relationship between UIC and MetS. Subsequently, the association between the UIC groups and MetS prevalence was detected using crude logistic regression. Multivariable logistic regression models were used to adjust for possible confounding factors. Univariate logistic regression analysis was performed to determine covariates associated with the MetS. The variables found to have moderate association $(P<0.10)$ with MetS in the univariate analysis were included in the final multivariate logistics regression models. However, the medical professional experience of physicians was equally important in selecting covariates. Some studies indicated that TSH 
levels and the presence of TPOAb and TgAb were related to metabolic disorders. ${ }^{12,13}$ Consequently, variables related to thyroid hormones were also included as covariates. All screened confounding factors were adjusted for in the multivariate logistics regression model to reduce bias. Two multivariate regression models were constructed to analyze the association in distinctive levels. We also performed tests for linear trends by entering the median value of each UIC category as a continuous variable in the models. Using STATA (version 15.1. StataCorp, USA), a two-tailed $P$ value $<0.05$ was considered statistically significant.

\section{Results}

\section{Clinical Characteristics of the Study Participants}

We recruited 2691 individuals (1315 males and 1376 females). According to the JIS standards, 809 participants were diagnosed with MetS (30.1\%). The median UIC level was 165.1 (IQR: $111.7-237.1 \mu \mathrm{g} / \mathrm{L}$ ), which is within the recommended range by the WHO (IQR: $100-199 \mu \mathrm{g} / \mathrm{L}$ ). Supplemental Table 1 shows the baseline characteristics of the study population. Clinical characteristics of participants in the MetS and control groups were also compared. Compared with individuals in the control group, those in the MetS group were older $(P<0.001)$; predominantly males and smokers; and had lower educational and income levels, and higher levels of BMI, LDL-C, TC, UA, TPOAb, and TgAb but lower UIC levels, with significant differences. However, TSH level and consumption of iodized salt had no significant differences between the groups $(P>0.05)$.

Characteristics of the study participants stratified by UIC are demonstrated in Table 1. Individuals were assigned to the following subgroups according to the corresponding UIC: iodine-deficient group (20.2\%), iodineadequate group (44.3\%), and high-iodine group (35.5\%). Compared with individuals in the iodine-deficient group, those in the high-iodine group were predominantly males, younger, and had higher education levels. Many of them were also current smokers and consumed iodized salt. Those indexes, including WC, DBP, TG, FPG, TC, LDL$\mathrm{C}$, and UA, showed an inverse linear trend with the UIC groups ( $P$ for trend $<0.05$ ). Although there was no noticeable trend in BMI among the groups, the BMI value in the high-iodine group, compared with those in the iodinedeficient groups, was significantly reduced $(P<0.01)$.
There was no discernible difference in the levels of HDL-C, TSH, TPOAb, and TgAb among the three UIC groups.

\section{Prevalence of MetS and Its Components in Different UIC Groups}

As shown in Table 2, MetS prevalence was inversely associated with the UIC groups, and this trend was more remarkable in males but almost diminished in females. The overall prevalence of MetS was $30.1 \%$, and high WC was the most frequent MetS component, followed by high BP, high TG levels, low HDL-C levels, and elevated FPG levels. When stratified according to UIC, the prevalence rates of MetS were $33.7 \%, 33.3 \%$, and $24.0 \%$ for the iodine-deficient, iodine-adequate, and highiodine groups, respectively. A negative trend was observed for MetS prevalence among the UIC groups, with a significant difference $(P<0.01)$. When analyzing the tendency of each component, the same pattern was observed for high WC, high TG, and low HDL-C levels. Subsequently, all individuals were grouped by sex in the subgroup analysis. A higher prevalence of MetS was observed in males than in females (34.1\% versus $26.2 \%$ ). The negative trend was also observed in the male subgroup for the prevalence of MetS and each component $(P<0.01)$. In the female subgroup, a comparison of MetS prevalence in the different UIC groups revealed the following results: high-iodine group $<$ iodine-deficient group $<$ iodine-adequate group. Only the prevalence of low HDL-C levels had a slightly inverse trend $(P=0.046)$. In females, MetS prevalence in the high-iodine group was significantly lower than that in the iodine-adequate group ( $P=0.004)$, but there was no difference compared with that in the iodine-deficient group $(P=0.143)$.

\section{MetS and UIC}

Logistics regression analysis was performed to reduce confounding factors and observe the relationship between iodine intake status and MetS prevalence. The results are shown in Table 3 and Figure 2. Using the iodine-adequate group as a reference, the odds ratios (ORs) of the risk of MetS in the iodine-deficient and high-iodine groups were calculated. Furthermore, an analysis was performed not only in the overall study population but also in the sexbased subgroups. Compared to the reference, individuals with a high-iodine status had a lower risk of MetS, with a crude OR of 0.63 (95\% CI 0.52-0.77) and adjusted ORs 
Table I Clinical Characteristics of Study Subjects in the Cross-Sectional Study

\begin{tabular}{|c|c|c|c|c|c|}
\hline & Total & UIC $<100$ & UIC 100-199 & UIC $\geq \mathbf{2 0 0}$ & \multirow[t]{2}{*}{$P$ for Trend } \\
\hline & $n=2691$ & $n=543(20.2 \%)$ & $n=I \mid 93(44.3 \%)$ & $\mathrm{n}=955(35.5 \%)$ & \\
\hline UIC (ug/L) & $165.10(111.70-237.10)$ & $7 I .3 I$ (52.4I-87.90) & $148.80(\mid 26.30-172.40)$ & $267.30(231.40-345.10)$ & $<0.00 I^{*}$ \\
\hline Male: N (\%) & 1315 (48.9\%) & $203(37.4 \%)$ & $604(50.6 \%)$ & 508 (53.2\%) & $<0.00 I^{*}$ \\
\hline Female: N (\%) & 1376 (5I.1\%) & $340(62.6 \%)$ & 589 (49.4\%) & 447 (46.8\%) & \\
\hline Age (years) & $43.43 \pm 15.88$ & $46.32 \pm 14.53$ & $44.38 \pm 15.62$ & $40.60 \pm 16.50$ & $<0.00 I^{*}$ \\
\hline High education: N (\%) & $990(36.8 \%)$ & $170(31.3 \%)$ & $4 I I(34.5 \%)$ & 409 (42.8\%) & $<0.001 *$ \\
\hline Income >5W: N (\%) & 1600 (59.5\%) & $329(60.6 \%)$ & 721 (60.4\%) & $550(57.6 \%)$ & 0.200 \\
\hline Smoking: N (\%) & 721 (26.8\%) & 105 (19.3\%) & $342(28.7 \%)$ & $274(28.7 \%)$ & $0.001 *$ \\
\hline lodine salt: N (\%) & 2097 (77.9\%) & $384(70.7 \%)$ & 912 (76.5\%) & 801 (83.9\%) & $<0.001 *$ \\
\hline Salt intake/day: N (\%) & & & & & 0.260 \\
\hline$<5 \mathrm{~g}$ & 222 (8.3\%) & 45 (8.3\%) & 90 (7.5\%) & 87 (9.1\%) & \\
\hline $5-10 \mathrm{~g}$ & $|82|(67.7 \%)$ & $376(69.2 \%)$ & 793 (66.5\%) & $652(68.3 \%)$ & \\
\hline$>10 \mathrm{~g}$ & $648(24.1 \%)$ & $122(22.5 \%)$ & $310(26.0 \%)$ & $216(22.6 \%)$ & \\
\hline BMI (kg/m2) & $24.19 \pm 3.74$ & $24.10 \pm 3.65$ & $24.43 \pm 3.79$ & $23.94 \pm 3.71$ & 0.110 \\
\hline$W C(\mathrm{~cm})$ & $82.93 \pm 10.11$ & $82.78 \pm 9.82$ & $83.62 \pm 10.01$ & $82.14 \pm 10.28$ & $0.040^{*}$ \\
\hline $\mathrm{SBP}(\mathrm{mmHg})$ & $125.02 \pm 17.09$ & $125.10 \pm 18.27$ & $125.30 \pm 17.23$ & $124.47 \pm 16.19$ & 0.480 \\
\hline $\mathrm{DBP}(\mathrm{mmHg})$ & $73.45 \pm 10.74$ & $73.53 \pm 11.02$ & $73.82 \pm 10.67$ & $72.95 \pm 10.67$ & $0.020^{*}$ \\
\hline TG (mmol/L) & I.I7 (0.78-I.77) & I.I8 (0.82-1.85) & $1.24(0.83-1.90)$ & I.03 (0.73-I.57) & $<0.00 I^{*}$ \\
\hline FBG (mmol/L) & $5.39 \pm 1.37$ & $5.48 \pm 1.63$ & $5.44 \pm 1.44$ & $5.28 \pm 1.06$ & $0.010^{*}$ \\
\hline HDL-C (mmol/L) & $1.40 \pm 0.42$ & $\mathrm{I} .4 \pm 0.42$ & $1.38 \pm 0.4 \mathrm{I}$ & $\mathrm{I} .4 \mathrm{I} \pm 0.42$ & 0.517 \\
\hline TC $(\mathrm{mmol} / \mathrm{L})$ & $4.60 \pm 1.12$ & $4.64 \pm 1.16$ & $4.67 \pm 1.13$ & $4.49 \pm 1.07$ & $<0.00 I^{*}$ \\
\hline LDL-C (mmol/L) & $2.71 \pm 0.79$ & $2.72 \pm 0.81$ & $2.75 \pm 0.78$ & $2.65 \pm 0.77$ & $0.010^{*}$ \\
\hline $\mathrm{UA}(\mu \mathrm{mol} / \mathrm{L})$ & $303.87 \pm 94.25$ & $292.78 \pm 96.91$ & $311.59 \pm 95.60$ & $300.52 \pm 90.19$ & 0.080 \\
\hline TSH (mlU/L) & $2.34(1.70-3.28)$ & $2.34(1.78-3.23)$ & $2.30(1.65-3.20)$ & $2.43(1.72-3.40)$ & 0.200 \\
\hline TPOAb (IU/mL) & $8.77(6.42-12.60)$ & $8.9(6.29-13.58)$ & $8.83(6.48-12.62)$ & $8.6(6.36-12.05)$ & 0.190 \\
\hline $\operatorname{TgAb}(\mathrm{IU} / \mathrm{mL})$ & $11.79(10.00-15.17)$ & II.77 (10.00-15.25) & $11.52(10.00-15.21)$ & $12.15(10.00-15.10)$ & 0.250 \\
\hline
\end{tabular}

Note: $* p<0.05$.

Abbreviations: UIC, urinary iodine concentration; BMI, body mass index; WC, waist circumference; SBP, systolic blood pressure; DBP, diastolic blood pressure; TG, triglyceride; FPG, fasting plasma glucose; HDL-C, high-density lipoprotein cholesterol; TC; total cholesterol; LDL-C, low-density lipoprotein cholesterol; UA, serum uric acid; TSH, thyroid-stimulating hormone; TPOAb, thyroid peroxidase antibody; TgAb, thyroglobulin antibody.

(aORs) of 0.7 (95\% CI 0.57-0.86, model 1) and 0.75 (95\% CI 0.6-0.95, model 2). Although the risk of MetS relatively increased in the iodine-deficient group, there was no significant difference. We performed tests for linear trends by entering the median value of each UIC group as a continuous variable in the models. As shown in Table 3, there was a significant inverse linear correlation between the risk of MetS and UIC in the total population
$\left(\begin{array}{ll}P & <0.01\end{array}\right)$. This trend was observed among males $(P<0.05)$ but not in females $(P$ for trend $>0.05)$. Among males, those in the high-iodine group had a lower risk of MetS (crude OR 1.47, $P=0.018$; aOR 1.46, $P=0.024$, model 1; aOR 1.36, $P=0.107$, model 2) and those in the iodine-deficient group had a higher risk of MetS (crude OR 0.6, $P<0.001$; aOR $0.67, P=0.003$, model 1; aOR $0.82, P=0.192$, model 2 ). After adjustment according to 


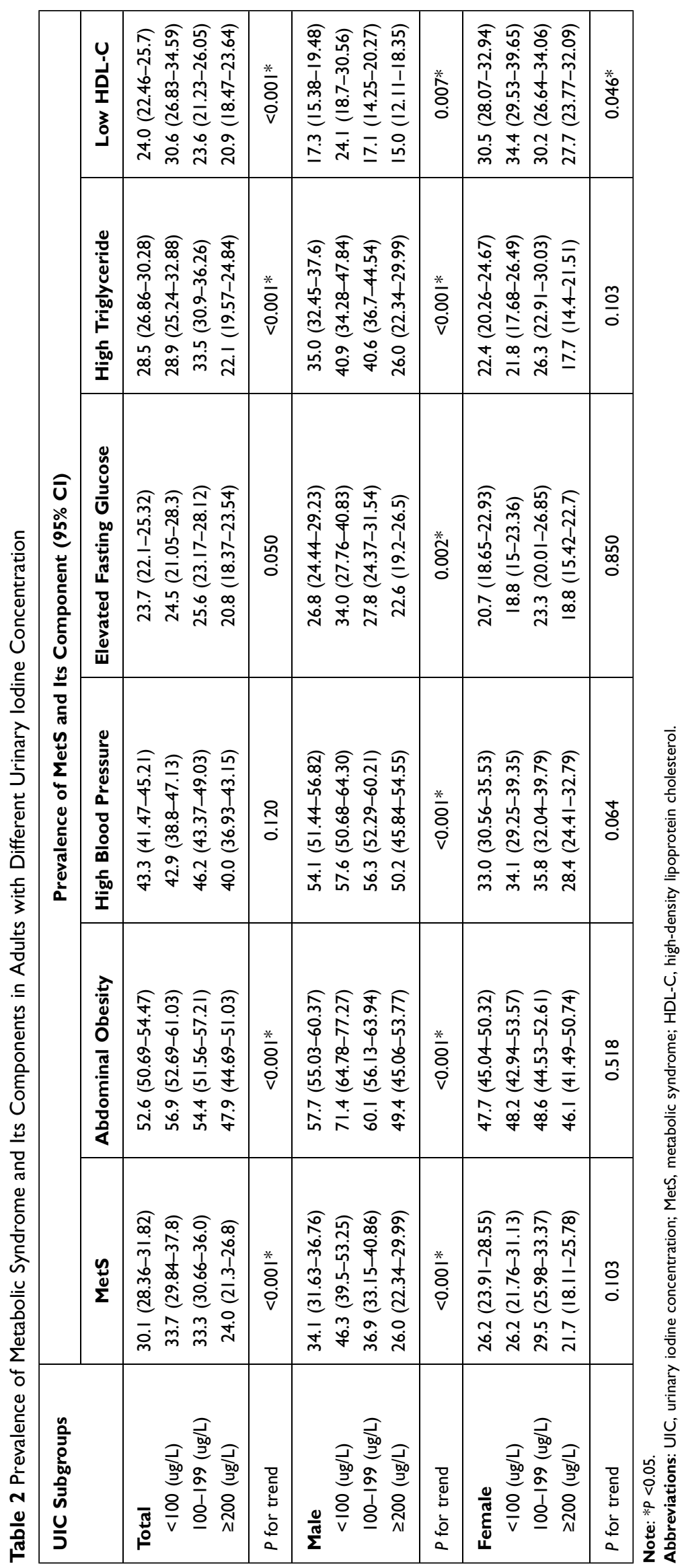


Table 3 Risk of Metabolic Syndrome in Adults with Different Urinary lodine Concentration

\begin{tabular}{|c|c|c|c|c|c|c|}
\hline \multirow{2}{*}{$\begin{array}{l}\text { UIC Subgroups (ug/ } \\
\text { L) }\end{array}$} & \multicolumn{2}{|c|}{ Crude Model } & \multicolumn{2}{|c|}{ Model I } & \multicolumn{2}{|c|}{ Model 2} \\
\hline & OR $(95 \% \mathrm{Cl})$ & $P$ & OR (95\% Cl) & $P$ & OR (95\% Cl) & $P$ \\
\hline \multicolumn{7}{|l|}{ Total } \\
\hline$<100$ & $1.02(0.82-1.26)$ & 0.862 & $1.04(0.82-1.30)$ & 0.768 & I.II (0.85-I.44) & 0.452 \\
\hline $100-199$ (ref) & I & - & I & - & 1 & - \\
\hline$\geq 200$ & $0.63(0.52-0.77)$ & $<0.001 *$ & $0.70(0.57-0.86)$ & $0.001 *$ & $0.75(0.60-0.95)$ & $0.018^{*}$ \\
\hline$P$ for trend & $P<0.00 I^{*}$ & & $P<0.00 I^{*}$ & & $0.004^{*}$ & \\
\hline \multicolumn{7}{|l|}{ Male } \\
\hline$<100$ & $1.47(1.07-2.03)$ & $0.018^{*}$ & $1.46(1.05-2.04)$ & $0.024 *$ & $1.36(0.93-1.99)$ & 0.107 \\
\hline $100-199$ (ref) & 1 & - & I & - & 1 & - \\
\hline$\geq 200$ & $0.60(0.46-0.78)$ & $<0.001 *$ & $0.67(0.51-0.87)$ & $0.003^{*}$ & $0.82(0.60-1.1 \mathrm{I})$ & 0.192 \\
\hline$P$ for trend & $P<0.001$ & & $P<0.001$ & & $0.014 *$ & \\
\hline \multicolumn{7}{|l|}{ Female } \\
\hline$<100$ & $0.85(0.63-1.14)$ & 0.273 & $0.80(0.57-I . I I)$ & 0.186 & $0.93(0.63-1.35)$ & 0.693 \\
\hline $100-199$ (ref) & I & - & I & - & I & - \\
\hline$\geq 200$ & $0.66(0.45-0.88)$ & $0.005^{*}$ & $0.72(0.52-0.99)$ & $0.049 *$ & $0.70(0.48-1.02)$ & 0.064 \\
\hline$P$ for trend & 0.060 & & 0.438 & & 0.130 & \\
\hline
\end{tabular}

Notes: Model I adjusted confounding factors included sex, age, menopause status, income, and education. Model 2 adjusted confounding factors included sex, age, menopause status, income, education, BMI, TC, LDL-C, UA, TSH, TPOAb, and TGAb. For male participants, menopause status was excluded from model 2. P presents the $P$ value for each OR. $P$ for trend: Test for linear trend of OR based on entering the median value of each category of UIC as a continuous variable in the models. $* P<0.05$. Abbreviations: UIC, urinary iodine concentration; OR, odds ratio; $\mathrm{Cl}$, confidence interval; ref, reference.

model 2, there was no significant difference between the groups. The relationship between iodine intake status and the risk of MetS in females was somewhat distinct, as the risk of MetS was reduced in the other two groups compared with that in the iodine-adequate group. However, the high-iodine group had the lowest risk of MetS (crude OR $0.66, P=0.005$; aOR $0.72, P=0.049$, model 1 ; aOR 0.7 , $P=0.064$, model 2).

\section{Discussion}

In this study, we investigated the prevalence of MetS in a moderate iodine intake area and the relationship between iodine intake status and the prevalence of MetS. The overall prevalence of MetS was 30.1\%, which was lower than that reported by the 2010 China non-communicable disease surveillance. ${ }^{4}$ The median UIC in the MetS group was lower than that in the control group. After grouping according to UIC, the prevalence of MetS gradually decreased with the ordered UIC groups, which indicated an inversely linear association between the two factors. Despite the fact that this trend varied by sex, the high-iodine group having the lowest prevalence of MetS and its components, was consistent. In our study, potential confounding factors, including sociodemographic factors, metabolic parameters, and thyroid-related indicators, were considered and adjusted as covariates in the multivariate logistic regression analysis. Logistic regression analysis performed using different regression models and analysis of population showed somewhat distinct results. After adjusting for sociodemographic variables, metabolic parameters, and thyroid-related index, compared with individuals in the iodine-adequate group, the OR of incident MetS in the high-iodine group reduced by $25 \%$, with a significant difference, while that in the iodine-deficient group increased by $11 \%$, with no significant difference. The risk of MetS significantly increased in the iodine-deficient group only among males, which indicated that males would more likely gain benefits from increased iodine intake, while females may require a higher amount of iodine intake to benefit from its protective effect. All the data presented above indicate a potentially protective role of high iodine intake against MetS development.

MetS is characterized by a constellation of interrelated risk factors for CVD and T2D. We observed decreased prevalence rates of high $\mathrm{WC}$, high $\mathrm{TG}$ levels, and low HDL-C levels, with increasing iodine intake, which was consistent with the findings of previous investigations. ${ }^{16,22,23}$ We also found that, with an increase in iodine intake, TC and LDL-C levels gradually 


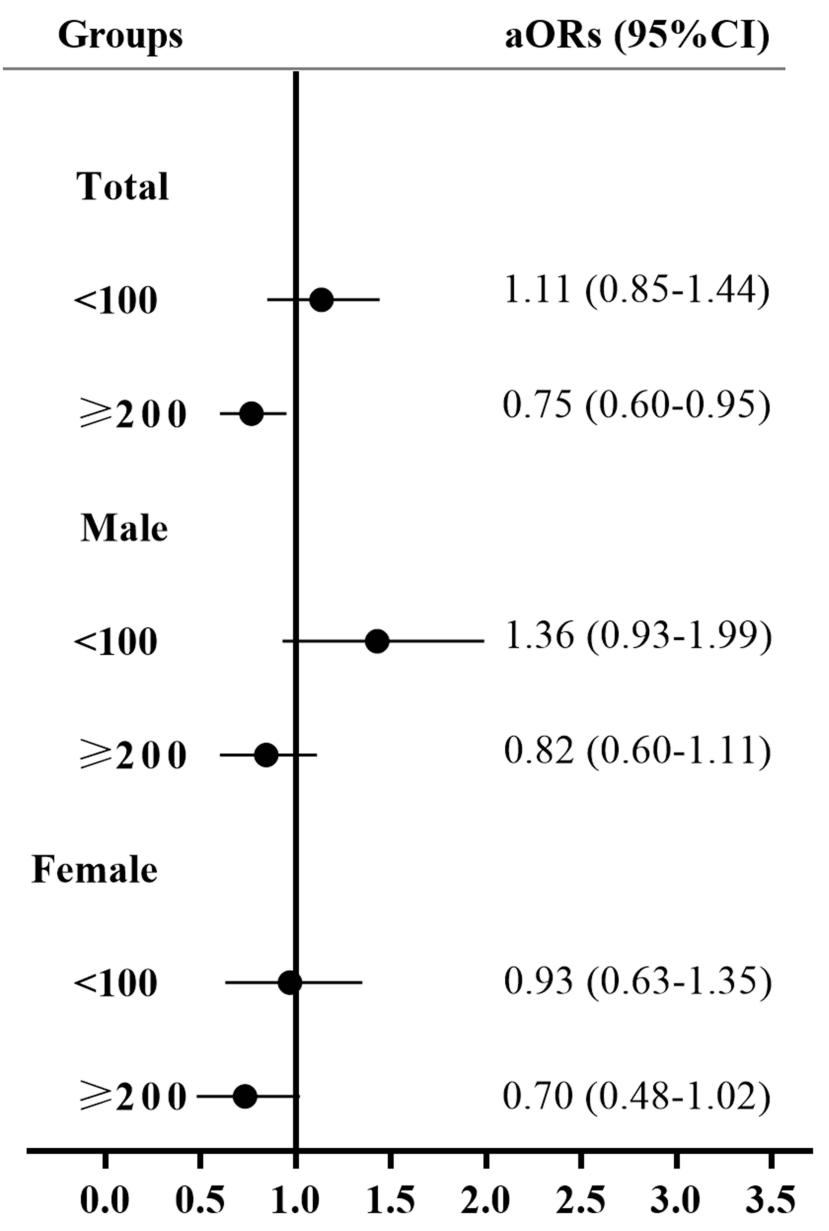

Figure 2 Risk of metabolic syndrome among different UIC groups when compared with the iodine-adequate group.

decreased. Above all, individuals with good dietary iodine status had a lower probability of having dyslipidemia. A randomized controlled trial of iodine supplementation in overweight women reported that iodine supplementation could reduce the prevalence of hypercholesterolemia. ${ }^{23}$ Additionally, a survey completed in the United States in 2016 confirmed that low UIC level was associated with increased odds for dyslipidemia, ${ }^{16}$ consistent with our research.

Although the trend tests of high BP and elevated FPG levels were not significant in our study; however, the highiodine group had the lowest prevalence rates of the MetS components Quantitative analysis demonstrated that, with increasing UIC levels, DBP gradually decreased, but SBP showed no significant change. The declined difference between groups was limited, and it could not achieve the threshold against positive high BP. We speculated that this maybe the same reason for FPG. Nevertheless, several studies reported that UIC was significantly associated with maternal BP and that iodine deficiency was a risk factor for the hypertensive disease of pregnancy and preeclampsia. ${ }^{24,25}$ Further research is necessary to confirm these findings.

An inverse relationship was observed between iodine intake status and the prevalence of MetS in this study. A prospective study of Korean menopausal women demonstrated that the average iodine intake showed a negative linear association with the incidence of MetS, ${ }^{22}$ which is similar to our results. According to these data, we could infer that adequate iodine status is likely to exert a protective effect against MetS and its components. Iodine is a crucial ingredient in the synthesis of thyroid hormones, which are important for multiple metabolic pathways such as glucose metabolism, lipolysis, and body weight regulation. Our study showed that iodine status is a crucial relevant factor for thyroid diseases, ${ }^{26}$ which could partly elucidate this appearance. However, the beneficial effect of iodine status on the regulation of metabolism occurred probably due to thyroid hormones and other pathways. Previous studies confirmed that the presence of thyroid autoantibodies and thyroid dysfunction was a confirmatory finding for dyslipidemia, hyperglycemia, central obesity, and MetS, ${ }^{27-29}$ even in the euthyroid population. $^{11}$

By far, the most widely accepted theory was that insulin resistance and chronic inflammation status appeared to have been at the core of the pathophysiology of the MetS. ${ }^{30,31}$ Therefore, dietary iodine intake may exert a protective effect on MetS development through the aforementioned pathogenesis. Several clinical trials reported that a low UIC correlated with insulin resistance, dyslipidemia, and MetS. ${ }^{23,32}$ The benefit that increasing iodine intake could alleviate MetS and dysmetabolism and even reduce the risk was observed in clinical trials ${ }^{22,25,33}$ and also in animal experiments. ${ }^{14}$ In vitro islet cell culture experiments demonstrated that iodine amplified glucose-induced firstand second-phase insulin secretion and reduced the lag time before the initiation of insulin secretion. ${ }^{34}$ Another in vitro experiment demonstrated that iodine supplementation significantly modified the expression of peroxisome proliferator-activated receptors, ${ }^{35}$ which were initially associated with the regulation of lipid metabolism, which is one of the nuclear receptors playing a central role in metabolic diseases. Additionally, it is well established that iodine exerts anti-inflammatory and antioxidant properties. Previous studies have shown that iodine is an effective antioxidant, and iodine could reduce free radicals as an 
electron donor, decrease organ damage by neutralizing radical oxygen species, and enhance the general antioxidant status in human serum. ${ }^{36}$ Moreover, marine algae as iodinerich food offset inflammation through several mechanisms: inhibition of pro-inflammatory enzymes such as COX-2 and iNOS, modulation of MAPK pathways, and NK-kB activation. ${ }^{37}$ Third, iodine exerts effects on metabolism by modulating the gut microbiome. Intake of iodine and dried red seaweed could affect the gut microbiome of rats, related to hosting metabolic regulations. ${ }^{14}$

The present study showed that people with high UICs appeared to have a lower risk of MetS, which differed after stratifying by sex. A gradual decrease in OR from the iodinedeficient group to the high-iodine group could be observed in the overall population and among males, suggesting a decreasing trend in the risk of MetS in respondents with increasing UICs. However, a significant reduction in the risk of MetS was observed only among females in the high-iodine intake group, indicating that females might need to consume more iodinecontaining diets to gain metabolic benefit. The potential reasons for this difference are as follows. First, the proportion of current smokers was significantly distinct by sex. The smoking rate among males was higher than that among females, which increases with the ordered UIC groups. Several studies showed that the levels of serum lipids and soluble immune/inflammatory markers were affected by smoking status. ${ }^{38}$ After adjustment for potential confounding factors, the protective effect of high-iodine intake against MetS was not significant. This could be due to the interaction effects of smoking with metabolic parameters and inflammatory markers that counteract the protective effect of iodine status. Second, hormonal differences between males and females. Estrogen was considered to confer protection owing to its beneficial effect on metabolism and the antioxidant function. An increased prevalence of MetS was observed in postmenopausal females, compared with that in premenopausal females, ${ }^{39}$ which could be partially confirmed. Meanwhile, a multicenter cohort study indicates that dietary iodine and seaweed consumption in postmenopausal women could be negatively associated with the prevalence of MetS and metabolic disorders. ${ }^{22}$ Third, the decreased sample size in each group after stratification by sex could have also caused the difference. Among all participants, after entirely adjusting for factors according to model 2, compared with that in the reference group, the risk of MetS in the high-iodine group reduced by $25 \%$, which remained significant. However, the underlying mechanisms remain mostly unknown, and future investigations are required.

However, this study also had some limitations. Since our study excluded pregnant women and minors, it could not represent the overall distribution of metabolic disorders in the population. Moreover, due to the cross-sectional nature of this study, we could not identify causality between iodine status and MetS. Considering all, further prospective cohort studies are required for assessing the relationship between iodine status and MetS.

\section{Conclusions}

This study suggests an inverse association between dietary iodine intake and MetS development. Achieving a more-thanadequate iodine status is a potential protective factor against the incidence of MetS and is somewhat distinct in the sex subgroups. Males are more likely to benefit from increased iodine intake, while females need to achieve a more-thanadequate iodine status to gain metabolic benefits. Moreover, more researches on iodine status and Mets are warranted to elucidate their relationship and underlying mechanisms better.

\section{Author Contributions}

All authors contributed to data analysis, drafting or revising the article, have agreed on the journal to which the article will be submitted, gave final approval of the version to be published, and agree to be accountable for all aspects of the work. Jing Zhao and Yinbiao Su are co-first authors.

\section{Funding}

The Outstanding Clinical Discipline Project of Shanghai Pudong [grant number PWYgy2018-03], the Health Scientific Research Project of Pudong New District Health Commission [grant number PW2019D-12], Medical and Health Program of Pudong New Area Science and Technology Development Fund [grant number PKJ2017-Y38], the Project of Shanghai Medical Key Specialty [grant number ZK2019C09], and Thyroid Clinical Research Center of Shanghai Health Medical College [grant number 20MC20200002].

\section{Disclosure}

The authors declare that they have no conflicts of interest for this work.

\section{References}

1. Alberti KG, Eckel RH, Grundy SM, et al. Harmonizing the metabolic syndrome: a joint interim statement of the International Diabetes Federation Task Force on Epidemiology and Prevention; National Heart, Lung, and Blood Institute; American Heart Association; World Heart Federation; International Atherosclerosis Society; and International Association for the Study of Obesity. Circulation. 2009;120(16):1640-1645. 
2. Esposito K, Chiodini P, Colao A, Lenzi A, Giugliano D. Metabolic syndrome and risk of cancer: a systematic review and meta-analysis. Diabetes Care. 2012;35(11):2402-2411. doi:10.2337/dc12-0336

3. Zimmet P, Alberti G, Kaufman F, et al. The metabolic syndrome in children and adolescents. Lancet. 2007;369(9579):2059-2061. doi:10.1016/S0140-6736(07)60958-1

4. Lu J, Wang L, Li M, et al. Metabolic syndrome among adults in China: the 2010 China noncommunicable disease surveillance. J Clin Endocrinol Metab. 2017;102(2):507-515.

5. Fenwick PH, Jeejeebhoy K, Dhaliwal R, et al. Lifestyle genomics and the metabolic syndrome: a review of genetic variants that influence response to diet and exercise interventions. Crit Rev Food Sci Nutr. 2019;59(13):2028-2039. doi:10.1080/10408398.2018.1437022

6. Taylor JY, Kraja AT, de Las Fuentes L, Stanfill AG, Clark A, Cashion A. An overview of the genomics of metabolic syndrome. J Nurs Scholarsh. 2013;45(1):52-59. doi:10.1111/j.1547-5069.2012.01484.x

7. Khalili M, Shuhart MC, Lombardero M, et al. Relationship between metabolic syndrome, alanine aminotransferase levels, and liver disease severity in a multiethnic North American cohort with chronic hepatitis B. Diabetes Care. 2018;41(6):1251-1259. doi:10.2337/dc18-0040

8. Velasco I, Bath SC, Rayman MP. Iodine as essential nutrient during the first 1000 days of life. Nutrients. 2018;10(3):290. doi:10.3390/ nu 10030290

9. Zimmermann MB, Boelaert K. Iodine deficiency and thyroid disorders. Lancet Diabetes Endocrinol. 2015;3(4):286-295. doi:10.1016/S2213-8587(14)70225-6

10. Leung AM, Braverman LE. Consequences of excess iodine. Nat Rev Endocrinol. 2014;10(3):136-142. doi:10.1038/nrendo.2013.251

11. Chen Y, Zhu C, Chen Y, et al. Are thyroid autoimmune diseases associated with cardiometabolic risks in a population with normal thyroid-stimulating hormone? Mediators Inflamm. 2018;2018:1856137. doi:10.1155/2018/1856137

12. Mehran L, Amouzegar A, Azizi F. Thyroid disease and the metabolic syndrome. Curr Opin Endocrinol Diabetes Obes. 2019;26 (5):256-265. doi:10.1097/MED.0000000000000500

13. Zhang J, Gao Y, Li Y, et al. The presence of serum TgAb suggests lower risks for glucose and lipid metabolic disorders in euthyroid general population from a national survey. Front Endocrinol. 2020;11:139. doi:10.3389/fendo.2020.00139

14. Shen H, Han J, Li Y, et al. Different host-specific responses in thyroid function and gut microbiota modulation between diet-induced obese and normal mice given the same dose of iodine. Appl Microbiol Biotechnol. 2019;103(8):3537-3547. doi:10.1007/s00253-019-09687-1

15. Rosique C, Lebsir D, Benatia S, et al. Metabolomics evaluation of repeated administration of potassium iodide on adult male rats. Arch Toxicol. 2020;94(3):803-812. doi:10.1007/s00204-020-02666-w

16. Lee KW, Shin D, Song WO. Low urinary iodine concentrations associated with dyslipidemia in US adults. Nutrients. 2016;8 (3):171. doi:10.3390/nu8030171

17. Karakaya RE, Saka M, Ozdemir D. Determining the relationship between dietary iodine intake, urinary iodine excretion and thyroid functions in people with type 2 diabetes mellitus. Arch Endocrinol Metab. 2020;64(4):383-389.

18. Al-Attas OS, Al-Daghri NM, Alkharfy KM, et al. Urinary iodine is associated with insulin resistance in subjects with diabetes mellitus type 2. Exp Clin Endocrinol Diabetes. 2012;120(10):618-622. doi:10.1055/s-0032-1323816

19. Liu J, Liu L, Jia Q, Zhang X, Jin X, Shen H. Effects of excessive iodine intake on blood glucose, blood pressure, and blood lipids in adults. Biol Trace Elem Res. 2019;192(2):136-144. doi:10.1007/ s12011-019-01668-9

20. Fleiss JL, Levin B, Paik MC. Statistical Methods for Rates and Proportions. 3th ed. New York: John wiley \& sons; 2013.

21. World Health Organization. Assessment of Iodine Deficiency Disorders and Monitoring Their Elimination: A Guide for Programme Managers. 3rd ed. Geneva: World Health Organization; 2007.
22. Park JK, Woo HW, Kim MK, et al. Dietary iodine, seaweed consumption, and incidence risk of metabolic syndrome among postmenopausal women: a prospective analysis of the Korean Multi-Rural Communities Cohort Study (MRCohort). Eur J Nutr. 2021;60 (1):135-146. doi:10.1007/s00394-020-02225-0

23. Herter-Aeberli I, Cherkaoui M, El Ansari N, et al. Iodine supplementation decreases hypercholesterolemia in iodine-deficient, overweight women: a randomized controlled trial. $J$ Nutr. 2015;145 (9):2067-2075. doi:10.3945/jn.115.213439

24. Gulaboglu M, Borekci B, Delibas I. Urine iodine levels in preeclamptic and normal pregnant women. Biol Trace Elem Res. 2010;136 (3):249-257. doi:10.1007/s12011-009-8539-y

25. Cuellar-Rufino S, Navarro-Meza M, García-Solís P, Xochihua-Rosas I, Arroyo-Helguera O. Iodine levels are associated with oxidative stress and antioxidant status in pregnant women with hypertensive disease. Nutr Hosp. 2017;34(3):661-666. doi:10.20960/nh.460

26. Wang B, He W, Li Q, et al. U-shaped relationship between iodine status and thyroid autoimmunity risk in adults. Eur J Endocrinol. 2019;181(3):255-266. doi:10.1530/EJE-19-0212

27. Laclaustra M, Moreno-Franco B, Lou-Bonafonte JM, et al. Impaired sensitivity to thyroid hormones is associated with diabetes and metabolic syndrome. Diabetes Care. 2019;42(2):303-310. doi:10.2337/ dc18-1410

28. Biondi B, Kahaly GJ, Robertson RP. Thyroid dysfunction and diabetes mellitus: two closely associated disorders. Endocr Rev. 2019;40 (3):789-824.

29. Chang CH, Yeh YC, Caffrey JL, Shih SR, Chuang LM, Tu YK. Metabolic syndrome is associated with an increased incidence of subclinical hypothyroidism - a Cohort Study. Sci Rep. 2017;7 (1):6754. doi:10.1038/s41598-017-07004-2

30. Cornier MA, Dabelea D, Hernandez TL, et al. The metabolic syndrome. Endocr Rev. 2008;29(7):777-822.

31. Russo B, Picconi F, Malandrucco I, Frontoni S. Flavonoids and insulin-resistance: from molecular evidences to clinical trials. Int J Mol Sci. 2019;20(9):2061. doi:10.3390/ijms20092061

32. Lecube A, Zafon C, Gromaz A, et al. Iodine deficiency is higher in morbid obesity in comparison with late after bariatric surgery and non-obese women. Obes Surg. 2015;25(1):85-89. doi:10.1007/ s11695-014-1313-z

33. Tørris C, Småstuen MC, Molin M. Nutrients in fish and possible associations with cardiovascular disease risk factors in metabolic syndrome. Nutrients. 2018;10(7):952. doi:10.3390/nu10070952

34. Nuñez-Anita RE, Arroyo-Helguera O, Cajero-Juárez M, LópezBojorquez L, Aceves C. A complex between 6-iodolactone and the peroxisome proliferator-activated receptor type gamma may mediate the antineoplastic effect of iodine in mammary cancer. Prostaglandins Other Lipid Mediat. 2009;89(1-2):34-42. doi:10.1016/j.prostaglandins.2009.04.001

35. Wang YX. PPARs: diverse regulators in energy metabolism and metabolic diseases. Cell Res. 2010;20(2):124-137. doi:10.1038/ cr.2010.13

36. Winkler R, Griebenow S, Wonisch W. Effect of iodide on total antioxidant status of human serum. Cell Biochem Funct. 2000;18 (2):143-146.

37. Barbalace MC, Malaguti M, Giusti L, Lucacchini A, Hrelia S, Angeloni C. Anti-inflammatory activities of marine algae in neurodegenerative diseases. Int J Mol Sci. 2019;20(12):3061. doi:10.3390/ ijms20123061

38. Bentley AR, Sung YJ, Brown MR, et al. Multi-ancestry genome-wide gene-smoking interaction study of 387,272 individuals identifies new loci associated with serum lipids. Nat Genet. 2019;51(4):636-648.

39. Stefanska A, Bergmann K, Sypniewska G. Metabolic syndrome and menopause: pathophysiology, clinical and diagnostic significance. Adv Clin Chem. 2015;72:1-75. 


\section{Publish your work in this journal}

Diabetes, Metabolic Syndrome and Obesity: Targets and Therapy is an international, peer-reviewed open-access journal committed to the rapid publication of the latest laboratory and clinical findings in the fields of diabetes, metabolic syndrome and obesity research. Original research, review, case reports, hypothesis formation, expert opinion and commentaries are all considered for publication. The manuscript management system is completely online and includes a very quick and fair peer-review system, which is all easy to use. Visit http://www.dovepress.com/testimonials.php to read real quotes from published authors. 